\title{
INTEGRATED OPTIMIZATION MODEL FOR PRODUCTION PLANNING AND SCHEDULING WITH LOGISTICS CONSTRAINTS
}

\author{
Chen, Y. X. \\ School of Economics and Management, Harbin Engineering University, Harbin 150001, China \\ E-Mail: chenyxdingdang@hrbeu.edu.cn
}

\begin{abstract}
Production planning and the development of manufacturing production scheduling are two important operational management tasks. The results of these tasks will strongly influence the development of corporate profits, as well as the efficiency of utilization of resources. Considering the production scheduling problems in production plans can effectively avoid conflicts between the specific implementation production plan and the actual situations, and ultimately maximize resource utilization efficiency. This paper attempts to establish an integrated optimization model of production planning and scheduling with consideration of logistics constraints. A modified heuristic algorithm is proposed to solve this problem. In the final portion of this paper, a simulation analysis is given to demonstrate the outstanding advantage of the model and the algorithm, which gives further thought for the production planning.

(Received, processed and accepted by the Chinese Representative Office.)
\end{abstract}

Key Words: Production Planning, Scheduling, Logistics Capability, Integrated Optimization Model, Particle Swarm Optimization

\section{INTRODUCTION}

At present, the new round of technological and industrial revolution is rapidly developing worldwide. The challenges of this development are economic and production in a globalized market, the diversification and personalization of the product demand, an external changeable environment, complex collaborative relationships, etc. As for manufacturing, Computer Integrated Manufacturing System (CIMS) is an essential tool for enterprises to gain information. CIMS is a system that integrates computers, information, intelligence, and optimization of the manufacturing system based on CIM theory. It is integration with the manufacturing systems in the enterprise, such as information management systems, production management systems, manufacturing resource planning systems, computer-aided design systems, etc.

Production planning and scheduling problem (PPSP) are very important in CIMS, and play an essential role in the convergence of enterprise management and production control. The companies must respond very quickly, improve flexibility, eliminate process redundancy and errors, and improve efficiency in the changing market demand to maintain corporate dominance in the global competition, especially in the complex domestic and international economic situation.

Production planning and the development of manufacturing production scheduling are two essential operational management tasks. The results of these tasks will strongly influence the development of corporate profits, as well as the efficiency of utilization of resources. Production planning refers to some medium-term decisions, including production, inventory, and other products in enterprises with production capacity constraints. The objective of production planning is to reduce the related cost. Therefore, the production target and the inventory level of each production cycle in horizon planning are the solutions of the production plan. The time period of the production plan is usually between a few months to 
one year. Production scheduling aims to solve the question of how to move forward, which is the main decision-making step of production tasks of each cycle of production planning and job assignment for machine sorting. The time period of production scheduling is typically between a few days to a few weeks. Obviously, these two issues are closely related, and the solution of production planning program is to produce input problems of conditions scheduling. Meanwhile, the capacity constraints often affect the solutions of scheduling problems. Specifically, in some special production environment, production planning and scheduling cannot be separated. For example, in the mass production systems of flexible job shop (FJS), it is necessary to determine the quantities of the various products, as well as the appropriate allocation of processing equipment for each product and production scheduling. It is necessary to consider the production capacity constraints, and the production capacity is related to the assigned machine. When assigning the appropriate machine, it is necessary to know each batch processing time, which in turn is related to product volume and processing time unit of a variety of products. Therefore, production planning and scheduling in nature are interrelated, and it is difficult to distinguish the boundaries of production planning and scheduling [1].

However, the traditional production planning and scheduling method is formulated according to the "separate rule". First, the company makes the production plan, and then produces detailed scheduling according to the given production plan. Specifically, the company firstly develops an aggregate production planning (APP) in the production planning layer according to customer orders, demand forecasts and historical data. Then, APP is broken down into detailed master production schedule (MPS) in the overall level of production and inventory decisions to develop the final number of production and inventory levels of products in a whole range of months or years for a certain period of time in days or weeks units of time. Lastly, according to the arrangement of the main production plan, the production scheduling is carried out, and the operation sequence and the machine assignment of each working procedure of every cycle can then be derived [2].

The main shortcoming of this traditional hierarchical method is that the scheduling constraints are considered in the planning process, which allows for a possible failure to obtain a feasible solution for the production planning [2]. Therefore, a better method is to integrate production planning and scheduling optimization. Current research trends reflect an integrated optimization of the production planning and scheduling, which means that in the production planning production capacity constraints are considered as well as the detailed scheduling as a constraint condition. The research background is for adjusting the time and product type or product family processing sequence related to the nature of the production environment or job shop [3-5].

Integrated optimization of production planning and scheduling has become a focus in industrial production process operations management research [6-8], and this paper focuses on integrated production planning and scheduling optimization of discrete manufacturing. The simultaneous problem SLSSP and scheduling of lot-sizing is similar to the integration of production planning and scheduling. The lot sizing scheme is defined as the production of each product within a planning range; in particular, the number of products that are not interrupted during the continuous processing of each product on each machine. This kind of problem has been studied within the constraints of production capacity, and determines the production batch of each product and the order of processing of various products. However, the SLSSP model generally does not consider feasible production planning and scheduling conditions. In most studies, the production environment of a single machine, parallel machine and flow shop are studied in order to adjust the time and processing sequence [9-11].

Recently, there is some research on production scheduling [12], but there has not been much research on production planning and scheduling integration optimization under the 
complex job shop environment [2]. Lasserre establishes a model that also includes planning and scheduling attributes. The decomposition method proposed alternate iteration planning and scheduling, and proves that the algorithm can be limited through iterations to converge to a local optimal solution [13]. Paper [14] proposes a decomposition algorithm based on Lagrange Relaxation (LR), which can obtain the optimal solution of the problem in theory based on the research of Lasserre. However, with the increase in the size of the problem, the demodulation of the sub problem will involve more computational burdens, and it is difficult to apply to large-scale problems. Fandel et al. established a double-time multi-product multilevel model of job shop batch planning and scheduling of simultaneous optimization [15]. This model is nonlinear in nature, and includes three different forms of 0 - 1 integer variable, so that the model is very difficult to solve. Papers [13-15] are based on a classic job shop as the background, where each process is defined for each workpiece on a specified machine. In actual production, flexible job shop allows processing in step on any number of machines. As far as is known, only paper [16] studies the integrated optimization of production planning and scheduling in a flexible job shop, and it establishes a model of flexible job shop production planning and scheduling, and designs a genetic algorithm to resolve the model.

\section{PROBLEM DESCRIPTION}

In manufacturing field, some flexibility is needed for the production workshop in order to balance the peak season and off-season orders. Different types and specifications of products such as engines can be produced in the same production line, and each launch may have different processing routes, or different operations or different tools at various points in the production line.

The existing production plan and production scheduling are usually conducted separately. First, the production plan is made according to the orders, inventory and production capacity, and then the scheduling is arranged based on the actual production situation. However, it often happens that the production scheduling is not coordinated with the production plan. Therefore, it is necessary to consider the production scheduling into the production plan.

In addition, the order does not finish until it is shipped to the customer. If the logistics capability is insufficient or logistics scheduling is not optimal, the product will only be stored in the warehouse, which is a waste of the enterprise's resources. From the perspective of customers, although the customer order is submitted once, the process of production inventory consumption tends to be relatively stable. Therefore, a more flexible way of customer service can be supplied if production plans can be made according to logistics capability. It is a very important task to integrate the logistics capability and production scheduling with the production plan to maximize the benefits, minimize the costs, as well as attain the comprehensive goal of customer service.

The difference of this paper with other related research is:

a) Most of the existing research assumes that all the demands must be met in the same period, and delayed deliveries will result in high penalty cost. However, this model allows the same products to be produced in the different plan periods.

b) The existing literature on production planning and production scheduling does not consider the logistics capability, while this model takes the logistics capability as constraint conditions.

The data of this paper is collected from a heavy machinery equipment diesel engine production workshop. The workshop has a complete engine production line, and some jobs or links have multiple coordinate stations. Each station can process a model of engine, or processes a variety of models of engines. The production plan is made monthly, referred to as a planning cycle, and every day is a production cycle. The demands for each product of all the 
customers are known in each planning cycle. Multiple processes are involved in the production of engines, and each process may have one or more devices. The processing capacity for each device is known. Some machines can produce different types of products. However, these different types require an adjustment in the tools, materials or personnel, and these adjustments consume time and cost. Considering the logistics constraints, a reasonable production plan and production scheduling are needed, which makes the production tasks in each planning cycle allowing for proper overtime. The objective of production planning is to develop a variety of production of engine products to meet the demands of the customers, and minimize the production cost, inventory cost, logistics cost and minimize the penalty cost. The objective of production scheduling is to identify processing sequence of various products on the same device, as well as minimize device adjustment cost and overtime cost for distribution of each machine. The objective of model is to minimize the total costs within the feasible scheduling scheme.

\section{MATHEMATICAL MODEL}

The explanations of the symbols are as follows:

Table I: The explanations of the symbols.

\begin{tabular}{|c|c|c|}
\hline No. & Symbols & Explanation of symbols \\
\hline 1 & $P$ & The total number of the types of the products \\
\hline 2 & $M$ & The sum of the machines \\
\hline 3 & $T$ & The total number of the planning periods \\
\hline 4 & $i, j$ & The code of the product. $i, j=1, \ldots, N$ \\
\hline 5 & $p_{i}$ & The number of the processes in the production of $i$ product \\
\hline 6 & $r, s$ & The number of the process, $r, s=1, \ldots, p_{i}$ \\
\hline 7 & $(i, r)$ & The $r$ process of $i$ product \\
\hline 8 & $m$ & The number of the machine, $m=1, \ldots, M$ \\
\hline 9 & $t$ & The number of the planning period, $t=1, \ldots, T$ \\
\hline 10 & $\overline{O I_{i}}$ & The initial inventory of the first production cycle of $i$ product \\
\hline 11 & $e_{i, r}^{m}$ & $\begin{array}{l}\text { The adjust time of }(i, r) \text { process on } m \text { machine (the adjustment time is } 0 \text { with no } \\
\text { adjustment) }\end{array}$ \\
\hline 12 & $f_{i, r}^{n}$ & The processing time of $(i, r)$ process on $m$ machine \\
\hline 13 & $g_{i, r}^{m}$ & $\begin{array}{l}\text { Decision variable, } g_{i, r}^{m}=1 \text { means that } m \text { machine is needed in the production, } \\
\text { otherwise, } g_{i, r}^{m}=0\end{array}$ \\
\hline 14 & $H(m)$ & The set of the process on the $m$ machine, virtual processes are not included \\
\hline 15 & $H B(m)$ & The set of the process on the $m$ machine, virtual product $H_{0}$ is included \\
\hline 16 & $H F(m)$ & The set of the process on the $m$ machine, virtual product $H_{p+1}$ is included \\
\hline 17 & $M(i, r)$ & The set of machines which are available for the $(i, r)$ process \\
\hline 18 & $c p_{i, t}$ & The unit product cost of $i$ product in the $t$ production cycle \\
\hline 19 & $c f_{i, t}$ & The unit punish cost for not meet the demand of $i$ product in the $t$ production cycle \\
\hline 20 & $c g_{i, t}$ & The preparation cost of $(i, r)$ process on $m$ machine \\
\hline 21 & $c h_{i, r}^{m}$ & The adjust cost of $(i, r)$ process on $m$ machine \\
\hline 22 & $c q_{m, t}$ & The unit over time cost on $m$ machine \\
\hline 23 & $c t_{i, t}$ & The unit distribution cost of $i$ product in the $t$ production cycle \\
\hline 24 & $d_{i, t}$ & The demand of $i$ product in the $t$ production cycle \\
\hline 25 & $\operatorname{lmax}_{i}$ & The celling amount of the production batch of $t$ production \\
\hline 26 & $\operatorname{lmin}_{i}$ & The floor amount of the production batch of $t$ production \\
\hline 27 & $\sigma$ & The overtime limit scale factor \\
\hline 28 & $W_{m, t}$ & The capability of $m$ machine in the $t$ production cycle \\
\hline 29 & $t \max _{i, t}$ & The maximum distribution amount of $i$ product in the $t$ production cycle \\
\hline
\end{tabular}


Table I (continuation)

\begin{tabular}{|c|c|l|}
\hline 30 & $\operatorname{Tmin}_{i, t}$ & The minimum distribution amount of $i$ product in the $t$ production cycle \\
\hline 31 & $q_{i, t}$ & The amount of the shortfall of $i$ product in the $t$ production cycle \\
\hline 32 & $x_{i, t}$ & The amount of the production of $i$ product in the $t$ production cycle \\
\hline 33 & $y_{i, t}$ & $\begin{array}{l}\text { Decision variable, } y_{i, t}=1 \text { means that } i \text { product is produced in } t \text { production cycle, } \\
\text { otherwise, } y_{i, t}=0\end{array}$ \\
\hline 34 & $z_{i, t}$ & $\begin{array}{l}\text { Decision variable, } z_{i, t}=1 \text { means that } i \text { product is delivered in } t \text { production cycle, } \\
\text { otherwise, } z_{i, t}=0\end{array}$ \\
\hline 35 & $B_{i, r}^{t}$ & The beginning time of $(i, r)$ process in $t$ production cycle \\
\hline 36 & $F_{i, r}^{t}$ & The finish time of $(i, r)$ process in $t$ production cycle \\
\hline 37 & $G_{m, t}$ & The final machining completion time on $m$ machine in $t$ production cycle \\
\hline 38 & $\alpha_{i, r}^{m, t}$ & $\begin{array}{l}\text { Decision variable, } \alpha_{i, r}^{m, t}=1 \text { means that }(i, r) \text { process is assigned on } m \text { machine in } t \\
\text { production cycle, otherwise, } \alpha_{i, r}^{m, t}=0\end{array}$ \\
\hline 39 & $\beta_{i, r, j, s}^{n, t}$ & $\begin{array}{l}\text { Decision variable, } \beta_{i, r, j, s}^{n, t}=1 \text { means that }(i, r) \text { process is before }(j, s) \text { process on } m \\
\text { machine is in } t \text { production cycle, otherwise, } \beta_{i, r, j,}^{n, t}=0\end{array}$ \\
\hline 40 & $O T_{m, t}$ & The overtime on $m$ machine in $t$ production cycle \\
\hline
\end{tabular}

In this paper, $H_{0}$ and $H_{p+1}$ are virtual products and the process of virtual product is defined as a virtual process. It is assumed that the number of the virtual processes is $M$, and all the related costs are a positive number $A$ which is large enough. The first processed virtual product is $H_{0}$ and the last processed virtual product is $H_{p+1}$ in each cycle.

$$
\begin{aligned}
& \min Z=\sum_{t=1}^{T} \sum_{i=1}^{P}\left(c f_{i, t} \cdot q_{i, t}+c p_{i, t} \cdot x_{i, t}+c g_{i, t} \cdot y_{i, t}+c t_{i, t} \cdot z_{i, t}\right)+\sum_{m=1}^{M} \sum_{t=1}^{T} \sum_{i=1}^{P} \sum_{r=1}^{p_{i}} c h_{i, r}^{m} \cdot \alpha_{i, r}^{m, l}+\sum_{m=1}^{M} \sum_{t=1}^{T} c q_{m, t} \cdot O T_{m, t} \eta(D, \dot{\gamma}) \\
& \text { s.t., } O I_{i}+q_{i, 1}+x_{i, 1}=d_{i, 1}, i=1, \cdots, P \\
& q_{i, t}+x_{i, t}=d_{i, t}, i=1, \cdots, P, t=2, \cdots, T \\
& \sum_{(i, r) \in H(m)}\left(e_{i, r}^{m} \cdot y_{i, t}+p_{i, r}^{m} \cdot x_{i, t}\right) \cdot \alpha_{i, r}^{m, t} \leq W_{m, t}+O T_{m, t}, m=1, \cdots, M, t=2, \cdots, T \\
& l \min _{i} \cdot y_{i, t} \leq x_{i, t} \leq l \max _{i} \cdot y_{i, t}, i=1, \cdots, P, t=2, \cdots, T \\
& t \min _{i} \cdot z_{i, t} \leq x_{i, t} \leq t \max _{i} \cdot z_{i, t}, i=1, \cdots, P, t=2, \cdots, T \\
& q_{i, t} \leq d_{i, t}, i=1, \cdots, P, t=2, \cdots, T \\
& \alpha_{i, r}^{m, t} \leq f_{i, r}^{m}, r=1, \ldots, p_{i}, i=1, \cdots, P, m=1, \cdots, M, t=2, \cdots, T \\
& F_{i, r}^{t} \geq B_{i, r+1}^{t}+\sum_{m \in M(i, r)}\left(e_{i, r}^{m} \cdot y_{i, t}+p_{i, r}^{m} \cdot x_{i, t}\right) \cdot \alpha_{i, r}^{m, t}, r=1, \ldots, p_{i}, i=1, \cdots, P, t=2, \cdots, T \\
& F_{i, r}^{t} \leq B_{i, r+1}^{t}, r=1, \ldots, p_{i}, i=1, \cdots, P, t=2, \cdots, T \\
& B_{i, r}^{t}+\left(e_{i, r}^{m} \cdot y_{i, t}+p_{i, r}^{m} \cdot x_{i, t}\right) \cdot \alpha_{i, r}^{m, t}-\left(1-\beta_{i, r, j, s}^{m, t}\right) \cdot A \leq B_{j, s}^{t}, r, s=1, \ldots, p_{i}, i, j=1, \cdots, P, \\
& m=1, \cdots, M, t=2, \cdots, T \\
& B_{j, s}^{t}+\left(e_{j, s}^{m} \cdot y_{j, t}+p_{j, s}^{m} \cdot x_{j, t}\right) \cdot \alpha_{j, s}^{m, t}-\left(1-\beta_{j, s, i, r}^{m, t}\right) \cdot A \leq B_{i, r}^{t}, r, s=1, \ldots, p_{i}, i, j=1, \cdots, P, \\
& m=1, \cdots, M, t=2, \cdots, T \\
& \sum_{m \in M(i, r)} \alpha_{i, r}^{m, t}=y_{j, t}, r=1, \ldots, p_{i}, i=1, \cdots, P, t=2, \cdots, T \\
& \sum_{(i, r) \in H B(m)} \beta_{i, r, j, s}^{m, t}=\alpha_{j, s}^{m, t}, m \in M(j, s), s=1, \ldots, p_{i}, j=1, \cdots, P, t=2, \cdots, T \\
& \sum_{(j, s) \in H F(m)} \beta_{i, r, j, s}^{m, t}=\alpha_{i, r}^{m, t}, m \in M(j, s), r=1, \ldots, p_{i}, i=1, \cdots, P, t=2, \cdots, T
\end{aligned}
$$




$$
\begin{gathered}
\sum_{(i, r) \in H(m)} \beta_{0, s, i, r}^{m, t} \leq 1, s=m, m=1, \cdots, M, t=2, \cdots, T \\
\sum_{(i, r) \in H F(m)} \beta_{i, r, 0, s}^{m, t}=0, s=m, m=1, \cdots, M, t=2, \cdots, T \\
\sum_{(i, r) \in H(m)} \beta_{i, r, p+1, s}^{m, t} \leq 1, s=m, m=1, \cdots, M, t=2, \cdots, T \\
\sum_{(i, r) \in H B(m)} \beta_{p+1, s, i, r}^{m, t}=0, s=m, m=1, \cdots, M, t=2, \cdots, T \\
\max _{m, t}\left\{F_{i, p_{i}}^{t}-\left(1-\beta_{j, s, i, r}^{m, t}\right) \cdot A\right\}, s=m, m=1, \cdots, M, t=2, \cdots, T \\
G_{\left(i, p_{i}\right) \in H(m)} \leq W_{m, t}+O T_{m, t}, m=1, \cdots, M, t=2, \cdots, T \\
0 \leq O T_{m, t} \leq \sigma \cdot W_{m, t}, m=1, \cdots, M, t=2, \cdots, T \\
B_{i, r}^{t}, F_{i, r}^{t}, O T_{m, t} \geq 0, x_{i, t} \geq 0, x_{i, t}^{m, t}, z_{i, t} \in\{0,1\}, i=1, \cdots, P, t=2, \cdots, T \\
\beta_{i, r, j, s}^{m, t} \in\{0,1\}, 0 \leq \sigma \leq 1, r, s=1, \cdots, p_{i}, i, j=1, \cdots, P, \\
m=1, \cdots, M, t=2, \cdots, T
\end{gathered}
$$

Eq. (1) is the objective function: minimize the total costs. Eqs. (2) and (3) are the constrains of materials balance. Eq. (4) is the constraint of the production capacity. Eq. (5) is the constraint of the batch production. Eq. (6) is the constrain of the logistics capability. Eq. (7) is the constraint of the shortfall of the products. Eq. (8) expresses that the process is only allowed to proceed on the available machines. Eqs. (9) and (10) are the constrains of the processing order. Eqs. (11) and (12) express that only one process is allowed in the same period for any one machine at the same time. Eq. (13) expresses that if the production is arranged, the process can only be on one machine at a given time. Eqs. (14) and (15) express that each process can only have a tight process before and after a tight process. Eqs. (16)-(19) express that virtual processes can only be processed at the beginning and the finish time on each machine. Eq. (20) refers to the completion time calculation method for each machine. Eq. (21) expresses that the machine completion time cannot exceed the available time. Eq. (22) refers to the overtime constraints. Eqs. (23) and (24) refer to the decision variable value constraints.

\section{QPSO ALGORITHM}

Particle Swarm Optimization (PSO) algorithm was first proposed in paper [17], which is a population-based searching method. Recently, some researches employ this algorithm to solve scheduling problems. However, this algorithm is highly affected by the initial values of the particle swarm, and it can't solve the large-scale problem very well, so this paper employ the quantum particle swarm optimization (QPSO) algorithm, which was proposed in paper [18].

The position of each particle is generated randomly and the velocity of particle is 0 in the first iteration. Then the position and velocity are updated by the following equations:

$$
\begin{gathered}
V_{i, j}(t+1)=w \cdot V_{i, j}(t)+c_{1} \cdot r_{1, i}(t) \cdot\left[P_{i, j}(t)-X_{i, j}(t)\right]+c_{2} \cdot r_{2, i}(t) \cdot\left[P_{g, j}(t)-X_{i, j}(t)\right] \\
X_{i, j}(t+1)=X_{i, j}(t)+V_{i, j}(t+1)
\end{gathered}
$$

According to the paper [19], in order to make sure the convergence, the particles should converge to attractor $C_{i}$ :

$$
C_{i, j}(t)=\left(c_{1} \cdot r_{1} \cdot P_{i, j}(t)+c_{2} \cdot r_{2} \cdot P_{g, j}(t)\right) /\left(c_{1} \cdot r_{1}+c_{2} \cdot r_{2}\right), j=1,2, L, d, c_{1}, c_{2}: U(0,1)
$$




$$
C_{i, j}(t)=\alpha \cdot P_{i, j}(t)+(1-\alpha) \cdot P_{g, j}(t), \alpha: U(0,1)
$$

where $\alpha=\left(c_{1} \cdot r_{1}\right) /\left(c_{1} \cdot r_{1}+c_{2} \cdot r_{2}\right), P_{i}$ and $P_{g}$ represent the personal and global best particles respectively.

The quantum state of a particle is depicted by a wave function $\psi(\vec{x}, t)[20]$. Then a global point can be derived in QPSO [21, 22]. The global point, mbest, is defined as the mean of the Pbest positions of all particles, and the equation is as follows:

$$
\text { mbest }(t)=\left(\text { mbest }_{1}(t), \text { mbest }_{2}(t), \mathrm{K} \text { mbest }_{d}(t)\right)=\left(\frac{1}{n} \sum_{i=1}^{n} P_{i, 1}(t), \frac{1}{n} \sum_{i=1}^{n} P_{i, 2}(t), \mathrm{K}, \frac{1}{n} \sum_{i=1}^{n} P_{i, d}(t)\right)
$$

The new position of a particle is:

$$
X_{i, j}(t+1)=C_{i, j}(t) \pm \beta \cdot \mid \text { mbest }_{j}-X_{i, j}(t) \mid \cdot \ln (1 / u), u=\operatorname{rand}(0,1)
$$

where $\beta$ denotes for the contraction-expansion coefficient.

The first step of QPSO algorithm is decoding. The array of decoding method is employed in this paper. Fig. 1 shows an array decoding example.

\begin{tabular}{cccccc|c|c|c|} 
Charge & 1 & 2 & $\ldots$ & $n$ & $\ldots$ & \multicolumn{1}{c}{$N$} \\
Cast & 1 & 3 & $\ldots$ & $k$ & $\ldots$ & 2 & 1 \\
\hline
\end{tabular}

Figure 1: Array decoding example.

In PPSP model, the array decoding method cannot cover all processes. Designed particle encoding and decoding method is as follows: the location of the particle refers to a processing task, and its length refers to the number of steps for machining task. The particle position of each dimension is rounded, and represents the processing steps used in the equipment respectively. For example, 1 means that the machine numbered 1 is used and 2 means that the machine numbered 2 is used. Since the available machines in each processing step may be different, the particle position for each dimension has a scope limit, and it can be stated by the set of the available machines for the processing tasks. For example, if the available machines number for a processing task is 3, then the value range for the position is from 1 to 7 . After particle movement, every dimension integer position of the particles can be identified according to the value of the result of the integer used equipment.

PPSP concrete implementation steps are as follows:

Step 1: initialization parameters and 20 particles are generated randomly. Particles of each dimension in particle location within the scope of the particle velocity in each dimension is between $0 \sim 1$.

Step 2: calculate the new position of each particle's fitness. Particle's fitness for the task completion time can be derived after decoding. The particles will be determined by using the equipment, and the task completion time can be calculated once the equipment is determined.

Step 3: update individual extremum and global extremum. The minimum completion time of each particle can be determined and then the individual extreme value can be updated base on the location of the minimum completion time. Then the minimum completion time can be found, and the global extrema based on all the particles with the particle position can be updated.

Step 4: update the position and speed, and then judge of the updated speed. If the speed is greater than the maximum speed, then it is considered as equal to the maximum speed. A maximum of one particle velocity for particle position is the width scope of each dimension.

Step 5: if the maximum number of iterations has been reached, it is over, otherwise go back to Step 2.

Step 6: end task scheduling. 


\section{SIMULATION ANALYSIS}

There are no standard tests in the existing research. Nor is there a database for the PPSP model proposed in this paper. Therefore, a proper deformation is needed for the scheduling problem of standard case to construct a series of suitable test examples for the PPSP model.

Five test examples are selected based on the papers [23-25], and new numerical examples are given: 4 products on 6 machines processing of $4 \times 6$ partly flexible JSP, $8 \times 8$ partly flexible JSP, $10 \times 6$ partly flexible JSP, $10 \times 10$ flexible JSP and $15 \times 10$ partly flexible JSP.

The parameters are set as follows: the demand of the product is $d_{i, t}=100, i=1, \ldots, P$, $t=1, \ldots, T$; the adjustment time is 50 times for the processing time, which means that $e_{i, r}^{m}=50 f_{i, r}^{n}$; if the batch production is $x_{i, t}=100$, then the batch processing time for each process is $100 f_{i, r}^{n}$, and the total time of batch processing time for each process is $150 f_{i, r}^{n}$. The available production capacity for each cycle, maximum normal production time is $W=150 \times C \max$, where $C \max$ is the optimal value for standard example of a single product of total completion time. Production adjustment cost is $c h f_{i, r}^{m}=150$; overtime limit scale factor is $\sigma=0.25$. According to the practical situation in days for production cycle, it is usually set to five days a week, 22 days a month, a quarter of 62 days. Therefore, the total production cycle is $T=5,22,62$. Then we can get a total of 15 sets of experiments, and 10 times calculation in each experiment. The experimental results are shown in Table II.

Table II: Calculation results based on the test function.

\begin{tabular}{|c|c|c|c|c|c|c|c|c|}
\hline \multirow{2}{*}{ No. } & Size & $\begin{array}{c}\text { Existing } \\
\text { reference }\end{array}$ & $\begin{array}{c}\text { Production } \\
\text { capacity }\end{array}$ & \multicolumn{5}{|c|}{ Results in this paper } \\
\cline { 2 - 9 } & $P \times M \times T$ & $C$ max & $W$ & $C$ best & $C$ mean & OTbest & OTmean & $\begin{array}{c}\text { Calculation } \\
\text { time }(\mathrm{s})\end{array}$ \\
\hline $\mathbf{1}$ & $4 \times 6 \times 5$ & 17 & 2550 & 2550 & 2600 & 0 & 50 & 23.46 \\
\hline $\mathbf{2}$ & $8 \times 8 \times 5$ & 14 & 2100 & 2100 & 2100 & 0 & 0 & 81.79 \\
\hline $\mathbf{3}$ & $10 \times 6 \times 5$ & 40 & 6000 & 6000 & 6020 & 0 & 20 & 284.36 \\
\hline $\mathbf{4}$ & $10 \times 10 \times 5$ & 7 & 1050 & 1050 & 1050 & 0 & 0 & 569.52 \\
\hline $\mathbf{5}$ & $15 \times 10 \times 5$ & 11 & 1650 & 1650 & 1650 & 0 & 0 & 1240.36 \\
\hline $\mathbf{6}$ & $4 \times 6 \times 22$ & 17 & 2550 & 2550 & 2550 & 0 & 0 & 95.84 \\
\hline $\mathbf{7}$ & $8 \times 8 \times 22$ & 10 & 2100 & 2100 & 2100 & 0 & 0 & 318.27 \\
\hline $\mathbf{8}$ & $10 \times 6 \times 22$ & 40 & 6000 & 6000 & 6000 & 0 & 0 & 126.44 \\
\hline $\mathbf{9}$ & $10 \times 10 \times 22$ & 7 & 1050 & 1050 & 1050 & 0 & 0 & 2280.07 \\
\hline $\mathbf{1 0}$ & $15 \times 10 \times 22$ & 11 & 1650 & 1650 & 1680 & 0 & 30 & 4561.25 \\
\hline $\mathbf{1 1}$ & $4 \times 6 \times 62$ & 17 & 2550 & 2550 & 2550 & 0 & 0 & 269.18 \\
\hline $\mathbf{1 2}$ & $8 \times 8 \times 62$ & 14 & 2100 & 2100 & 2100 & 0 & 0 & 953.27 \\
\hline $\mathbf{1 3}$ & $10 \times 6 \times 62$ & 40 & 6000 & 6000 & 6010 & 0 & 10 & 3746.97 \\
\hline $\mathbf{1 4}$ & $10 \times 10 \times 62$ & 7 & 1050 & 1050 & 1050 & 0 & 0 & 6034.92 \\
\hline $\mathbf{1 5}$ & $15 \times 10 \times 62$ & 11 & 1650 & 1650 & 1650 & 0 & 0 & 15569.46 \\
\hline
\end{tabular}

We take the first experiment for explanation. The first serial shows the number of experiments. The second column shows that the experiments include the groups with four products and six machines, and five consecutive production cycle calculations. The existing literature only considers when processing is an optimal production time for every product as given in the third column. The production capacity of each cycle is given in the fourth column, $W=150 \times C \max =150 \times 17=2550$, which means the time available is 2550 each cycle. The fifth column to the ninth column shows the calculation results of this paper. Table II shows that this algorithm has achieved good results in different backgrounds and different sizes of grouping experiments. 


\section{CONCLUSIONS}

In this paper, with the background of a heavy machinery equipment of diesel engine production workshop, an integrated optimization model of production planning and scheduling with consideration of logistics capability was established for the flexible job shop. The logistics capability, needs, loss and flexible factors such as the workpiece processing routes are considered in this model. An improved QPSO algorithm for solving the model is designed, and measures of improvement for reducing the limitations and weaknesses in the improved algorithm of PPSP solving speed and effect are proposed in view of the basic particle swarm algorithm. Considering the production scheduling problems in production plan can effectively avoid conflicts between the specific implementation production plan and the actual situations, and ultimately maximize resource utilization efficiency and enterprise benefit.

\section{ACKNOWLEDGMENT}

This research was partially supported by the Humanities and Social Science Foundation of Chinese Ministry of Education (NO.13YJC630015), the Heilongjiang Natural Science Foundation (NO.F201341) and the National Natural Science Foundation of China (NO.71101034).

\section{REFERENCES}

[1] Pinedo, M. L. (2005). Planning and Scheduling in Manufacturing and Services, Springer Science+Business Media, New York

[2] Maravelias, C. T.; Sung, C. (2009). Integration of production planning and scheduling: Overview, challenges and opportunities, Computers \& Chemical Engineering, Vol. 33, No. 12, 1919-1930, doi:10.1016/j.compchemeng.2009.06.007

[3] Xue, G.-S.; Offodile, O. F.; Zhou, H.; Troutt, M. D. (2011). Integrated production planning with sequence-dependent family setup times, International Journal of Production Economics, Vol. 131, No. 2, 674-681, doi:10.1016/i.ijpe.2011.02.012

[4] Qi, Y.-N.; Tang, M.-C.; Zhang, M. (2014). Mass customization in flat organization: The mediating role of supply chain planning and corporation coordination, Journal of Applied Research \& Technology, Vol. 12, No. 2, 171-181, doi:10.1016/s1665-6423(14)72333-8

[5] Kim, H.; Jeong, H.-I.; Park, J. (2008). Integrated model for production planning and scheduling in a supply chain using benchmarked genetic algorithm, The International Journal of Advanced Manufacturing Technology, Vol. 39, No. 11-12, 1207-1226, doi:10.1007/s00170-007-1298-Z

[6] Li, Z.-K.; Ierapetritou, M. G. (2009). Integrated production planning and scheduling using a decomposition framework, Chemical Engineering Science, Vol. 64, No. 16, 3585-3597, doi:10.1016/j.ces.2009.04.047

[7] Terrazas-Moreno, S.; Grossmann, I. E. (2011). A multiscale decomposition method for the optimal planning and scheduling of multi-site continuous multiproduct plants, Chemical Engineering Science, Vol. 66, No. 19, 4307-4318, doi:10.1016/j.ces.2011.03.017

[8] Shah, N. K.; Ierapetritou, M. G. (2012). Integrated production planning and scheduling optimization of multisite, multiproduct process industry, Computers \& Chemical Engineering, Vol. 37, 214-226, doi:10.1016/j.compchemeng.2011.08.007

[9] Quadt, D.; Kuhn, H. (2009). Capacitated lot-sizing and scheduling with parallel machines, backorders, and setup carry-over, Naval Research Logistics, Vol. 56, No. 4, 366-384, doi: $10.1002 /$ nav.20345

[10] James, R. J. W.; Almada-Lobo, B. (2011). Single and parallel machine capacitated lotsizing and scheduling: New iterative MIP-based neighborhood search heuristics, Computers \& Operations Research, Vol. 38, No. 12, 1816-1825, doi:10.1016/j.cor.2011.02.005

[11] Ramezanian, R.; Saidi-Mehrabad, M.; Teimoury, E. (2013). A mathematical model for integrating lot-sizing and scheduling problem in capacitated flow shop environments, The 
International Journal of Advanced Manufacturing Technology, Vol. 66, No. 1-4, 347-361, doi:10.1007/s00170-012-4329-3

[12] Aydemir, E.; Koruca, H. I. (2015). A new production scheduling module using priority-rule based genetic algorithm, International Journal of Simulation Modelling, Vol. 14, No. 3, 450-462, doi:10.2507/IJSIMM14(3)7.299

[13] Lasserre, J. B. (1992). An integrated model for job-shop planning and scheduling, Management Science, Vol. 38, No. 8, 1201-1211, doi:10.1287/mnsc.38.8.1201

[14] Xiong, R.; Chen, H.-X.; Hu, B.-S. (1996). An integration model for production planning and job shop scheduling and its Lagrangian relaxation-based solution approach, Journal of Xidian University (Natural Science), Vol. 23, No. 4, 71-78

[15] Fandel, G.; Stammen-Hegene, C. (2006). Simultaneous lot sizing and scheduling for multiproduct multi-level production, International Journal of Production Economics, Vol. 104, No. 2, 308-316, doi:10.1016/j.ijpe.2005.04.011

[16] Zhang, X.-D.; Yan, H.-S. (2005). Integrated optimization of production planning and scheduling for a kind of job-shop, The International Journal of Advanced Manufacturing Technology, Vol. 26, No. 7-8, 876-886, doi:10.1007/s00170-003-2042-y

[17] Kennedy, J.; Eberhart, R. C. (2001). Swarm Intelligence, Morgan Kaufman Publishers, San Francisco

[18] Jin, X.; Liang, Y.-Q.; Tian, D.-P.; Zhuang, F.-Z. (2013). Particle swarm optimization using dimension selection methods, Applied Mathematics and Computation, Vol. 219, No. 10, 51855197, doi:10.1016/j.amc.2012.11.020

[19] Liu, J.; Sun, J.; Xu, W.-B. (2006). Quantum-behaved particle swarm optimization with adaptive mutation operator, Jiao, L.; Wang, L.; Gao, X.-B.; Liu, J.; Wu, F. (Eds.), Advances in Natural Computation, LNCS, Vol.. 4221, 959-967, doi:10.1007/11881070_126

[20] Tang, D.-Y.; Cai, Y.-M.; Zhao, J.; Xue, Y. (2014). A quantum-behaved particle swarm optimization with memetic algorithm and memory for continuous non-linear large scale problems, Information Sciences, Vol. 289, 162-189, doi:10.1016/j.ins.2014.08.030

[21] Sun, J.; Xu, W.-B.; Feng, B. (2004). A global search strategy of quantum-behaved particle swarm optimization, 2004 IEEE Conference on Cybernetics and Intelligent Systems, 111-116, doi: $10.1109 /$ iccis.2004.1460396

[22] Yang, Z.; Qiu, H.-L.; Luo, X.-W.; Shen, D. (2015). Simulating schedule optimization problem in steelmaking continuous casting process, International Journal of Simulation Modelling, Vol. 14, No. 4, 710-718, doi:10.2507/IJSIMM14(4)CO17

[23] Zhang, C.-S.; Yang, Q.-Y.; Sun, J.-G.; Zheng, L.-H. (2009). A hybrid algorithm for flowshop scheduling problem, Acta Automatica Sinica, Vol. 35, No. 3, 332-336, doi: $\underline{10.3724 /}$ sp.j.1004.2009.00332

[24] Zhang, G. H. (2009). Research on methods for flexible job shop scheduling problems, Huazhong University of Science and Technology, Wuhan

[25] Xia, W.-J.; Wu, Z.-M. (2005). An effective hybrid optimization approach for multi-objective flexible job-shop scheduling problems, Computers \& Industrial Engineering, Vol. 48, No. 2, 409-425, doi:10.1016/j.cie.2005.01.018 